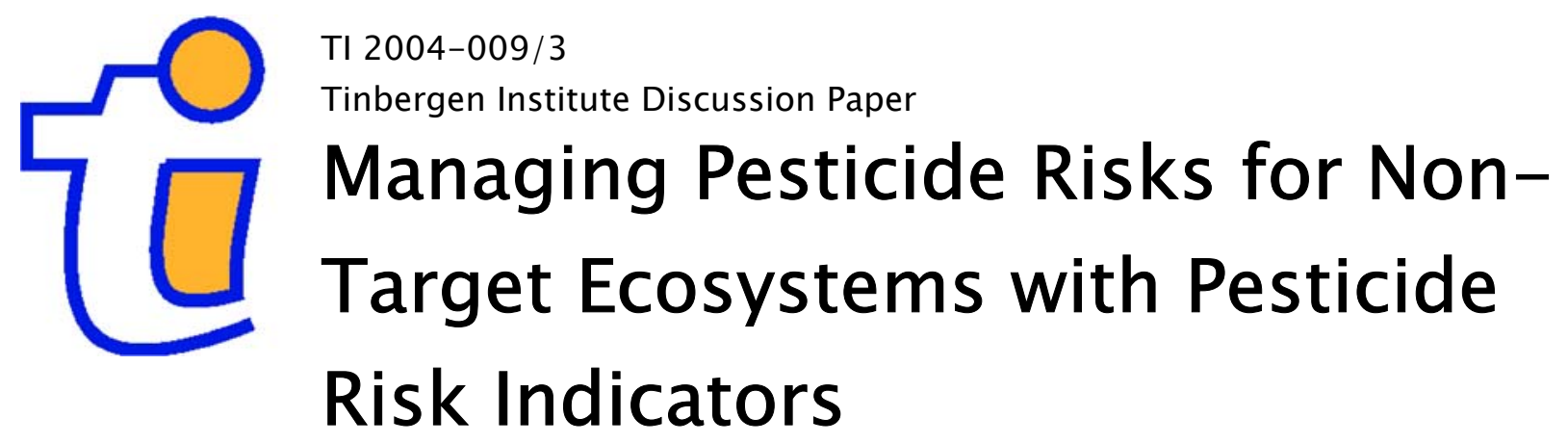

Chiara Maria Travisja,b

Peter Nijkampc

Marco Vighid

Paolo Giacomellib

a Fondazione Eni Enrico Mattei, b University of Milano, Italy, c Vrije Universiteit Amsterdam, and Tinbergen Institute, d University of Milano-Bicocca, Italy. 


\section{Tinbergen Institute}

The Tinbergen Institute is the institute for economic research of the Erasmus Universiteit Rotterdam, Universiteit van Amsterdam, and Vrije Universiteit Amsterdam.

Tinbergen Institute Amsterdam

Roetersstraat 31

1018 WB Amsterdam

The Netherlands

Tel.: $\quad+31(0) 205513500$

Fax: $\quad+31(0) 205513555$

Tinbergen Institute Rotterdam

Burg. Oudlaan 50

3062 PA Rotterdam

The Netherlands

Tel.: $\quad+31(0) 104088900$

Fax: $\quad+31(0) 104089031$

Please send questions and/or remarks of nonscientific nature to driessen@tinbergen.nl.

Most TI discussion papers can be downloaded at http://www.tinbergen.nl. 


\title{
Managing Pesticide Risks for Non-Target Ecosystems with Pesticide Risk Indicators: a Multicriteria Approach
}

\author{
Chiara Maria Travisi ${ }^{\mathrm{a}, \mathrm{b}}$, Peter Nijkamp $^{\mathrm{c}}$, Marco Vighi ${ }^{\mathrm{d}}$ and Paolo Giacomelli ${ }^{\mathrm{b}}$ \\ (a) Fondazione Eni Enrico Mattei, C.so Magenta, 63 \\ 20123 - Milan, Italy \\ Phone: +39-02-520.36951 \\ Fax $+39-02-520.36946$ \\ (b) University of Milano \\ Department of Agricultural and Environmental Economics and Policy, Via Celoria, 2 \\ 20133 - Milano, Italy \\ (c) Free University \\ Department of Spatial Economics, De Boelelaan 1105 \\ 1081 HV Amsterdam, the Netherlands \\ (d) University of Milano-Bicocca \\ Department of Environmental Sciences, P.zza della Scienza, 1 \\ 20126 - Milan, Italy
}

\begin{abstract}
In the search for effective pesticide risk management tools, the design of pesticide risk indicators is nowadays receiving increasing attention as a complement to more established regulatory strategies. In the present paper, we apply some recently developed pesticide risk indices and test their potential for management purposes. A pilot approach is proposed, which explores pesticide worst-case hazard scenarios at different space-time scales by means of a set of 5 ecotoxicological risk indices. The results are then interpreted from the perspective of a decision support method using the so-called Critical Threshold Value approach. Our risk analysis is then enriched within a multicriteria framework which integrates environmental, agronomic and economic objectives.
\end{abstract}

Key words: pesticides; hazard and risk assessment; risk indices; decision support tools; multicriteria analysis. 


\section{Introduction}

Since the late 1970's a wealth of scientific research from different disciplines has shed light on the off-farm human health and environmental risks ${ }^{1}$ of pesticide use; it has provided evidence that an indiscriminate use of chemical inputs in the agricultural production system would have not been environmentally or socially sustainable in the long run. This new awareness has prompted OECD countries to design and implement a variety of programmes and policies to reduce risks associated with pesticide use. For a detailed synthesis we refer to [17]. After two decades of research activity in the OECD countries, the findings from various pesticide risk scenarios are still contradictory. Due to the historically human-driven rather than environmentally-driven background of pesticide risk management in the past decades, which have led to a situation where potential pesticide risks to human health (general population and agricultural workers) can be considered to be reasonably under control, at present the dimension and nature of pesticide ecological effects is still largely unknown. Recent scientific insights highlight the problem of potential environmental side-effects of plant protection products (to control pests or weeds) on sensitive species and habitats, the so-called non-target organisms and ecosystems ${ }^{2}$.

In the search for effective tools to manage pesticide risk for non-target ecosystems, a broad agreement is arising in the scientific community on the usefulness of pesticide risk indicators as instruments capable to achieve a meaningful compromise between the demand for a sound scientific approach and the need for transparent public policy tools [18] [19].

This paper examines the role of risk indicators as a complement to more established policy instruments. A case study assessment of the applicability of risk indices within a decision support

\footnotetext{
${ }^{1}$ In risk assessment the terms 'hazard' and 'risk' are used to describe human health and environmental effects. Hazard is a function of toxicity and exposure; it demonstrates a potential. Risk is the assessment of the actual risk, which is the potential (or the probability) of the hazard to actually occur, given a biotic system exposed to it. In this paper, both the terms effect and risk refer to hazard.

${ }^{2}$ The term non-target organism indicates all the living organisms, with the only exception of the pests, which are specifically intended to be killed by pesticide applications. Non target ecosystems are those that, even if not directly treated, can be reached and spoilt by pesticides. Natural processes of environmental diffusion (run-off, drift, volatilization, bioaccumulation, etc.) can mobilize chemicals from the area of application to other non-target compartments, so that pesticide effects can spread -at different space-time scales- beyond local boundaries. As a consequence, surface water, ground
} 
system is presented. In the reminder of the paper, Section 2 discusses the basic principles and challenges in risk indices design. Section 3 then introduces the empirical case study, while Section 4 presents some risk assessment results. In Section 5, the outcomes from our risk assessment analysis are put in the context of a decision support method, while Section 6 presents and discusses main findings.

\section{Towards Risk Management Tools: Pesticide Risk Indicators}

The vivid debate on the design of pesticide risk indicators is based on the stance of their assumed complementarity to more consolidated and standardised procedures, such as risk assessment and registration (see [17]). From this perspective, it is an intriguing challenge to strengthen the definition and development of new policy-relevant tools, providing government officials and stakeholders with additional sound scientific and user-friendly support. Indicators would neither be used to substitute existing procedures nor to quantify pesticide risks in a strict sense; rather, they are expected to help national regulatory institutions to estimate general trends in pesticide risk reduction and to judge the effectiveness of their programmes.

The final report of the first OECD “Workshop on Pesticide Risk Indicators" [19] emphasizes that indicators may be designed for different purposes which will determine how much a sophisticated methodology is required, and how much and what types of data are needed. This argument is essential to guarantee that any interpretation of the meaning of indicators is consistent with the knowledge required, and that the indicators' results are not misinterpreted or employed beyond their proper contests.

On a first level of assessment, risk indicators may be designed as instruments for predictive risk management approaches, to offer preliminary insights into the status quo of pesticide risks. They may be developed to obtain baseline information about pesticide use and risks, focusing on one or more realistic hazardous scenarios, and they may guide the identification of potential trouble spots 
and vulnerable areas where risk reduction might be requisite. A proper design of risk indicators may also provide insights to compare, and eventually classify, several pesticide risks in respect to both the substance of concern -or mixture of substances- and the environmental target at stake. Finally, cumulative risks associated with the use of multiple pesticides may also be explored.

On a second level of action, indicators may be specifically designed for monitoring the impacts of pesticides policies and programmes during their several stages of implementation. In this case, indicators should be generated to track risk trends over time and space, which reflect also the dynamics in boundary conditions affecting pesticide risks ${ }^{3}$.

The previous arguments, though briefly discussed, suggest that challenges for future research are manifold. The first task is striking a compromise between scientific accuracy and decisionmaking pragmatism. Both experts and managers should be able to give a transparent interpretation of the information provided by indicators. Experts need to accurately interpret, reproduce and eventually refute results, whereas managers are asked to correctly interpret and use outcomes within the decision making process.

To satisfy such conditions, indicators should be consistent with modern principles of pesticide risk assessment and registration, thus combining information on pesticide hazard and exposure for each of the environmental compartments at risk (see [2], [3], and [4]) ${ }^{4}$. In this sense, a set of indicators, separately dealing with risks for different environmental targets, would be highly preferable to a single overall one. The use of sets of indicators would allow comparing results of scientific predictions on the severity of different pesticide risks with some a priori patterns of preferences/priorities, as expressed either by the risk-managers or by the stakeholders involved in the decision process. To some extent, the availability of sets of indicators -providing predictions for each of the endangered environmental compartments- would also enable the evaluation of risk/risk

\footnotetext{
treatments. Some studies mention that as much as $90 \%$ of the pesticides applied may or will reach non-target environments [5].

${ }^{3}$ To give an example, the time variable might be included as endogenous data in indicators by means of updating information on the level of pesticide use.

${ }^{4}$ This paper focuses on pesticide risks for non-target ecosystems; the discussion does not address risks to human health.
} 
and risk/benefit trade-offs, which are particularly relevant considering the multidimensionality of pesticide impacts.

To return to our main line of reasoning, whenever the indicators' design fulfils risk assessment principles, these instruments might help to develop plausible visions on negative side-effects of pesticide use at different time and spatial scales. Depending on the data and on the specifications set during the design process, the results may provide useful information into both risks for varying endangered compartments (surface water, ground water, epigean soil etc.), and risk scenarios at different space-time scales (short-term vs long-term horizons; local, regional or global scale). ${ }^{5}$

As already noted, the main challenge is the development of dynamic indicators including data on risk (hazard and exposure) and data on the conditions and quantity of pesticide use (amount applied per unit area, total area treated, total quantity of pesticide used, frequency of application, crop timing, formulation type etc.). Such a dynamic perspective would enable to measure the impacts of pesticide risk reduction programmes and policies, and to follow risk trends over time and space. Outcomes from a non-static analysis would provide intriguing visions on past and present experiences, giving important feedbacks for future risk reduction actions.

To conclude this section, some elements deserve a further comment. Firstly, indicators are crude measures of risks and should not be the sole basis for decision making. Risk trends and other information shown by indicators need to be confirmed and enriched by a closer investigation before regulatory or management action is taken. Besides, indicators are relative measures, not exact measures of real risk, and whether or not it is important for an indicator to correspond closely to real risk depends upon the purpose of the indicator and how it will be employed. In this sense, different indicators provide different results, and it is not yet possible to say which results are most accurate or which indicator provide the true information. Given the complexity of real-world

\footnotetext{
${ }^{5}$ One should not confuse the ability of indicators to reflect trends over time, i.e. a dynamic perspective, with the one of predicting risk scenarios at different time and spatial scales. In the former case, updating data on pesticide use is included in the indicators, thus allowing tracking risk trends over time. In the latter case, instead, the time dimension is not endogenous in the scenario, which gives a static picture of potential risks. Different scenarios can refer to varying time horizons, but they remain static visions referring to some fixed spots on the time axis.
} 
conditions and the intrinsic uncertainty of risk, the use of one -or of a combination of- various

alarm systems should always be preferred as this would lower the chance of missing the management of some non-negligible risks.

\section{Testing Risk Indexes: a Study on Herbicide Strategies in Italy}

In recent years, a number of pesticide risk indicators have been developed to provide information on the level of environmental hazard associated with pesticides by pre-specified criteria. The criteria defined to evaluate the acceptability of environmental risks are generally based on the concept of toxicity-exposure ratio (TER). ${ }^{6}$ This ratio should be calculated for each of the environmental non-target compartments at risk (surface water, ground water, soil) to establish critical threshold values as a trigger for the need for further investigations. On the other side, TERs can be used in comparative analysis and preliminary analysis of pesticide risks, with the introduction of adequate safety factors representing the limits of acceptable risk for each component of the non-target compartments.

In general, the proposed index-systems are founded on the development of a score for a set of physical-chemical, toxicological, and ecotoxicological properties of the substances considered (see [8], [12], and [21]). The scores are then combined through a proper algorithm in order to obtain a numerical expression of the level of potential risk related to the compound. Rating systems are usually based on standard environmental worst-case scenarios to guarantee the highest level of protection in accordance with the stance of the precautionary principle. Consequently, they assess the potential risk (or hazard) inborn into pesticides use. ${ }^{7}$

The relatively low complexity of this kind of approach, in respect to complete on-site risk

\footnotetext{
${ }^{6} \mathrm{~A}$ TER is defined as the ratio between a toxicological end-point (i.e. $\mathrm{LD}_{50}$, NOEL) and a Predicted Environmental Concentration (PEC): TER= (LD 50 or NOEL)/PEC. A PEC for a certain pollutant is the pollutant's concentration into a certain environmental compartment. Ideally, PECs should be measured in the environmental compartments of concern during monitoring campaigns, but -more often- PECs are predicted by mean of environmental diffusion modelling.

${ }^{7}$ Risk should not be confused with hazard (or potential risk). The 'hazard' of a pesticide is its potential to pose some negative effects on biological systems. There is a potential risk if predictions show that pesticide concentration in the environment exceeds the environmental quality criteria or risk based residue limits. Yet, for an assessment of the actual risk, the actual (real) exposure of a biological system has to be compared to concentrations known to actually exert negative effects.
} 
assessment procedures, as well as its predictive and comparative potential, suggests that it might be successfully employed to support and orient risk reduction strategies, beyond their use within registration procedures. Seen from this perspective, risk indices would find their natural setting in the preliminary stage of collection of information that precedes the definition and implementation of regulations. In this framework, their adoption might be particularly useful for the development of plausible visions on negative side-effects of pesticide use -at different space-time scales- and for the identification of patterns of priority for action. In a more advanced stage, the adoption of regional or local risk scenarios instead of standard worst-case scenarios would also allow a site-specific analysis with a direct connection to the relevant territory. On such conditions, the elaboration of decision-support tools for pesticide-oriented regional sustainability would represent an additional promising dimension of research development.

The focus of our paper is on the Italian context where rating systems for environmental pesticide risk have been recently developed -with analogous theoretical foundations- within a project sponsored by the Italian Environmental Protection Agency (ANPA). For a comprehensive description of principles and results of the ANPA project we refer to: [6], [7] and [8]. The usefulness of such tools for management purposes is tested here with an application to conventional agriculture in Italy. The analysis addresses herbicide use and explores herbicide worst-case hazard scenarios, at different space-time scales, for two of the most diffuse field crops: maize and rice. ${ }^{8}$ The procedure developed is illustrated in Figure 1. Step 1 implies the analysis of the Italian herbicide market -for maize and rice- and the identification of the main risk sources. Step 2 performs hazard assessments of risky agricultural practices and provides plausible visions upon different types of ecological impacts. Finally, in Step-3, the outcomes from Step-2 are enriched with additional information on agronomic and economic objectives, and risk scenarios are analysed

\footnotetext{
${ }^{8}$ Analogous analysis was also performed for other two important field crops: soybeans and field beet. For synthesis reasons, the paper illustrates only the case of maize and rice. In Italy, the herbicide market represents more than the $44 \%$ of the overall pesticide sector (insecticides $29 \%$; fungicides $21 \%$ ) [25]. The economic value of the herbicide sector reaches $40 \%$ of the total pesticides market. More than the $70 \%$ of the total amount of herbicides consumed is incorporated in the production of maize, wheat, rice and field beet, while fruit and vegetable farming absorbs the remaining part. Among the others, rice and field beet are the most herbicide-dependent farming practices [25].
} 
within the context of decision support methods.

Figure 1: Procedure for the analysis of hazard scenarios for non-target environments. Step-1 identifies major risk sources; Step-2 performs risk assessments of hazardous activities; Step-3 analyses risk scenarios in a decision support frame.

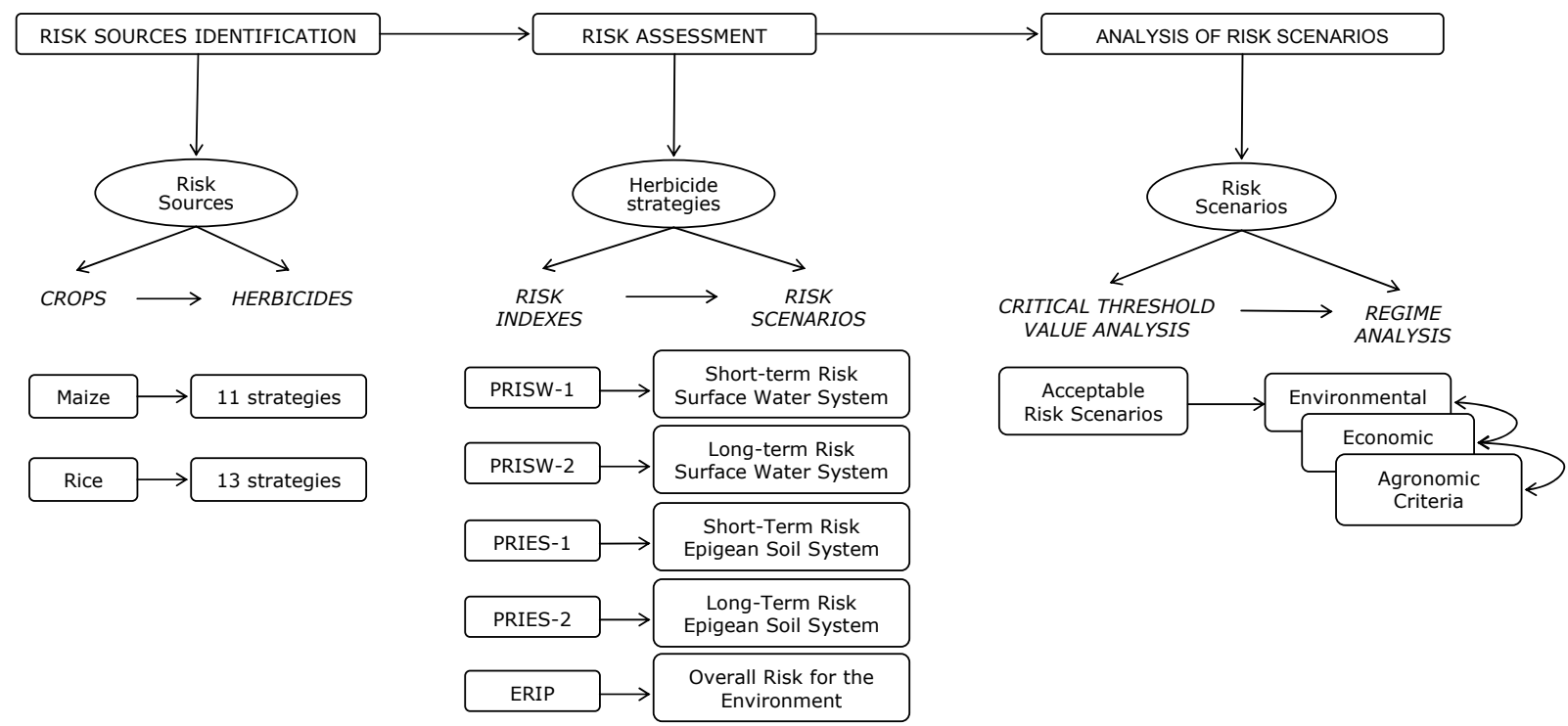

\section{Hazard Assessment}

Thanks to close cooperation with a group of Italian agronomists we could depict a realistic image of the potential ecological impacts attached to the herbicide practices employed in maize and rice production. For each crop of concern, we identified the herbicide strategies mostly used at a national level with similar agronomic action and purpose 9 . Overall, 24 strategies were selected, which typically implies the use of mixtures of two or more herbicide active ingredients ${ }^{10}$. Table 1 offers a summarized overview of the strategies ${ }^{11}$.

\footnotetext{
${ }^{9}$ For each crop type, the selected strategies are those that are applied to most of the national crop acreage; they have similar agronomic usage and are virtually mutually alternative to one another.

${ }^{10}$ Since crops can be treated at different stages of the vegetative cycle, the selected strategies include either pre-emergence and post-emergence herbicides (or a combination of the two).

${ }^{11}$ In maize production, the current trend is to adopt one single herbicide treatment per crop season, either in pre or post agronomic emergence. In Table 1, alternatives A to D are pre-emergence treatments while alternatives $\mathrm{E}$ to $\mathrm{M}$ refer to post emergence action. The peculiar environmental conditions of rice paddyfield are particularly suitable to the development of a complex vegetable biocenosis. Among such weed variety, those species
} 
Table 1: Sets of alternative herbicide strategies representative of maize and rice production.

\begin{tabular}{lll}
\hline MAIZE & \multicolumn{2}{l}{ RICE } \\
\hline & A. Tiocarbazil \\
A. Alachlor + Terbutilazine & B. Dimepiperate \\
B. Metolachlor + Terbutilazine & C. Dimepiperate + Molinate \\
C. Metolachlor + Pendimetalin & D. Tiobencarb \\
D. Isoxaflutol + Aclonifen & E. Tiobencarb + Molinate \\
E. Rimsulfuron + Prosulfuron + Primisulfuron & F. Tiobencarb + Propanile \\
F. Rimsulfuron + Dicamba & G. Molinate \\
G. Rimsulfuron + Terbutilazine & H. Quinclorac \\
H. Rimsulfuron + Fluroxipir & I. Propanile \\
I. Nicosulfuron + Dicamba & L. Pyrazoxyfen \\
L. Nicosulfuron + Sulcotrione & M. Oxadiazon \\
M. Nicosulfuron + Prosulfuron + Primisulfuron & N. Pretilaclor \\
& O. Azimsulfuron \\
\hline
\end{tabular}

To seek a correlation between herbicides use and the related risk of occurrence of negative sideeffects on environmental compartments, each strategy was characterised by means of 5 ecotoxicological risk indices assessing hazards for both aquatic and terrestrial non-target ecosystems ${ }^{12}$.

The indexes, developed in a project sponsored by the Italian Environmental Protection Agency ANPA, are entirely based on information required by Annex VI of the EU Directive 414/91/EEC for the registration and re-registration of plant protection products (Uniform Principles) ${ }^{13}$.

For surface water and epigean system two different time-spatial scales are taken into consideration. The short term (local scale) indices refer to a risk posed by a pesticide immediately after the on-field application (PRISW-1: Short-Term Pesticide Risk Index for Surface Water System; PRIES-1: Short-Term Pesticide Risk Index for Epygean Soil System). On the other side, two indices aim at evaluating pesticide risk on a medium time horizon and on an area beyond local boundaries (PRISW-2: Long-Term Pesticide Risk Index for Surface Water System; PRIES-2: Long-Term Pesticide Risk Index for Epygean Soil System). Finally, a comprehensive index evaluates the overall risks posed by pesticides to the environment (ERIP: Environmental Risk Index for Pesticides).

\footnotetext{
and ecotypes of monocotyledonous grass belonging to the genus Echinochloa represent the most diffuse and aggressive rice weeds. Selected alternative for rice are those acting against Echinocloa distribution in rice paddies.

${ }^{12}$ In the original ANPA project, three different environments were considered to provide a set of 7 indexes: surface water, terrestrial hypogean and terrestrial epigean systems. Due to the partial lack of basic toxicological data on the hypogean system, the present paper limits the analysis to surface water and epigean soil.

${ }^{13}$ Uniform Principles aim at standardising pesticides admission procedures in Europe, by providing a general outline and guidance of the effects evaluation. According to the Uniform Principles, all aspects of human health and the environment -including biota- have to be considered in the evaluation of fate, distribution and probable effects of pesticides.
} 
Consistently with modern risk assessment approaches, the indices integrate exposure parameters (rate of application, environmental distribution, bioaccumulation and soil persistence) and information on the effects $\left(\mathrm{EC}_{50}\right.$, NOEL) that pesticides can exert on a set of nontarget organisms considered as representative of each environmental system ${ }^{14}$.

The general procedure for the development of the indices consists of two sequential steps. Firstly, a PEC is calculated using simple dilution models or more complex models, based on the fugacity approach and specific for surface water inputs (Fugacity Level I by Mackay [13]; SoilFug: by Di Guardo [1]) ${ }^{15}$. Once a PEC is available, TERs are calculated by mean of toxicity data concerning relevant bioindicators. The algorithms assign sub-scores to each TER value, which are then weighted considering the ecological role of each bioindicator and its meaning in the overall risk evaluation. Finally, sub-scores are combined in a suitable algorithm to provide a final synthetic numerical outcome (see [6], [7] and [8]). For long-term and general indexes, due to difficulties in quantitatively calculating a PEC, the procedure may eventually be different. In this case, a scoring approach substitutes the PEC approach, which is used to convert fate variables (i.e., persistence, bioaccumulation etc.) into scores, subsequently combined into an overall score for exposure. When dealing with mixtures, the original algorithms have been modified under the hypothesis that all considered chemicals act with the same mode of action (in [10]: the Concentration Additional Model by Loewe, 1926) ${ }^{16}$.

Indexes can assume values ranging from 0 (virtually no risk) to 100 (maximum risk). Table 2

\footnotetext{
${ }^{14}$ Representative bioindicators are selected according to Directive 414/91/EEC. For surface water and epigean soil representative non-target organisms are, respectively: Algae, Daphnia magna, Fish; Plants, Bees, Beneficial arthropods, Birds, Mammals. The general index ERIP considers all previous bioindicators plus Earthworms. In case of lack of toxicity data, some default assumptions were used (for details see [8]).

${ }^{15}$ An exhaustive explanation of the environmental scenarios in which PECs are calculated is reported in [7].

${ }^{16}$ The unspecific narcotic effect of herbicides on non-target organisms justifies the adoption of such a stance. However, we are conscious that this approximation might increase the level of uncertainty of our risk predictions. Particularly, we expect that risk measures related to mixtures might be slightly overestimated here. Nevertheless, considering that the actual knowledge on mixture toxicity is still poor and that available data on pesticide use often underestimate the magnitude of this environmental problem, we believe that this bias might be tolerated here. The actual knowledge on mixtures' toxicity is still extremely slight and many efforts are needed for a complete understanding of the interaction mechanisms among substances. For mixtures, algorithms were modified as follows: for each $j$ th representative bioindicator, an overall toxicity-exposure ratio, denoted by TER ${ }_{\text {mixj }}$, substitutes TERs due to single substances. $\mathrm{TER}_{\text {mixj }}$ is calculated by adding the unit of toxicity $\left(\mathrm{TU}_{\mathrm{i}}\right)$ relative to each $i$ th component of the mixture, as follows:

$$
\mathrm{TER}_{\text {mix } \mathrm{j}}=1 / \sum_{\mathrm{i}}\left(1 / \mathrm{TU}_{\mathrm{i}}\right)
$$

where: $\mathrm{TU} i=(1 / \mathrm{TER} i) ; i=(1, . . \mathrm{n})$, with $\mathrm{n}=$ number of components of the mixture; $j=(1, . ., \mathrm{m})$, with $\mathrm{m}=$ number of representative bioindicators.
} 
illustrates risk scores as computed for the 24 herbicide treatments assessed ${ }^{17}$.

It goes without saying that managers and stakeholders might run into cognitive difficulties when dealing with scientific outcomes synthetically expressed as numerical scores. The ecotoxicological information synthesized by risk indexes, need to be converted into unequivocal terms. To overcome this problem, for each ecotoxicological index we developed a risk classification in which increasing scores correspond to more severe risk levels. Risks range from negligible to very high $^{18}$ (Table 2). Each risk level was then illustrated in qualitative terms providing a brief description of the possible impacts that experts ascribe to it. Different risk levels are therefore associated with plausible visions of potential environmental effects as illustrated in Table 2.

Table 2: Classes of risk and related potential negative impacts for each ecotoxicological risk indices.

\begin{tabular}{|c|c|c|}
\hline Risk Level & PRISW-1 & Potential Short-term Effects on Aquatic Ecosystem \\
\hline Negligible & $\leq 5$ & Negligible impacts \\
\hline Low & $>5-\leq 15$ & Moderate alteration of the aquatic biotic communities. \\
\hline Medium & $>15-\leq 40$ & Alteration of the aquatic biotic communities. Local dying out of most sensitive species of fishes and invertebrate. \\
\hline High & $>40-\leq 80$ & $\begin{array}{l}\text { Alteration of the aquatic biotic communities with reduction in sensible species of fishes and invertebrates. Potential reduction in } \\
\text { the community productivity. }\end{array}$ \\
\hline Very high & $>80$ & Serious effects on aquatic communities with a reduction in growth and productivity. Damping-off of fishes and invertebrates. \\
\hline Risk Level & PRISW-2 & Potential Long-term Effects on Aquatic Ecosystem \\
\hline Negligible & $\leq 5$ & Negligible impacts \\
\hline Low & $>5-\leq 10$ & Moderate alteration of the aquatic biotic communities. \\
\hline Medium & $>10 \leq 30$ & $\begin{array}{l}\text { Alteration of the aquatic biotic communities. Local dying out of most sensitive species of fishes and invertebrates; } \\
\text { bioaccumulation in the trophic chain. }\end{array}$ \\
\hline High & $>30 \leq 60$ & $\begin{array}{l}\text { Serious alteration of the aquatic biotic communities with changes in the existing species of fishes and invertebrates. Potential } \\
\text { reduction in the community productivity. Bioaccumulation in the trophic chain. }\end{array}$ \\
\hline Very high & $>60$ & Very serious effect on aquatic communities with a reduction in growth and productivity. Damping-off of fishes and invertebrates. \\
\hline
\end{tabular}

\footnotetext{
${ }^{17}$ The complete database and an electronic page for the calculation of the 5 indexes are available upon request to the authors.

${ }^{18}$ Risk classes were defined in light of results obtained from the indexes' validation procedure, performed within the ANPA project.
} 


\begin{tabular}{|l|l|l|}
\hline \multicolumn{1}{|c|}{ Risk Level } & \multicolumn{1}{|c|}{ PRIES-1 } & \multicolumn{1}{c|}{ Potential Short-term Effects on Terrestrial Ecosystem } \\
\hline Negligible & $\leq 5$ & Negligible impacts \\
\hline Low & $>5-\leq 15$ & Moderate alteration of terrestrial non-target communities \\
\hline Medium & $>15 \leq 50$ & $\begin{array}{l}\text { Alteration of terrestrial non-target communities: beneficial arthropods (pollinator insects and natural pests' antagonists), birds } \\
\text { and small mammals. Local migration of most sensitive species. }\end{array}$ \\
\hline High & $>50 \leq 70$ & $\begin{array}{l}\text { Serious alteration of population dynamics of terrestrial non-target species. Damping-off of most sensible arthropods, birds and } \\
\text { small mammals. }\end{array}$ \\
\hline Very high & $>70$ & $\begin{array}{l}\text { Very serious effects on terrestrial organisms with a reduction in growth and productivity. Significative increase of the death rate } \\
\text { of populations. }\end{array}$ \\
\hline
\end{tabular}

\begin{tabular}{|l|l|l|}
\hline \multicolumn{1}{|c|}{ Risk Level } & \multicolumn{1}{|c|}{ PRIES-2 } & \multicolumn{1}{c|}{ Potential Long-term Effects on Terrestrial Ecosystem } \\
\hline Negligible & $\leq 5$ & Negligible impacts \\
\hline Low & $>5-\leq 15$ & $\begin{array}{l}\text { Moderate alterations of terrestrial non-target communities: beneficial arthropods (pollinator insects, natural pests' antagonists), } \\
\text { birds and small mammals. }\end{array}$ \\
\hline Medium & $>15 \leq 40$ & Alteration of terrestrial non-target communities. Local migration of most sensitive species. Bioaccumulation in the trophic chain. \\
\hline High & $>40 \leq 70$ & $\begin{array}{l}\text { Serious alteration of population dynamics of terrestrial non-target species. Damping-off of most sensible arthropods, birds and } \\
\text { small mammals. Bioaccumulation in the trophic chain. }\end{array}$ \\
\hline Very high & $>70$ & $\begin{array}{l}\text { Very serious effects on terrestrial organisms with a reduction in growth and productivity. Significative increase of the death rate } \\
\text { of populations. Bioaccumulation in the trophic chain. Effects on bird populations might extend beyond local boundaries. }\end{array}$ \\
\hline
\end{tabular}

\begin{tabular}{|l|l|l|}
\hline \multicolumn{1}{|c|}{ Risk Level } & ERIP & \multicolumn{1}{|c|}{ Potential Effects on General Environment } \\
\hline Negligible & $\leq 10$ & Negligible impacts \\
\hline Low & $>10-\leq 25$ & Low probability of alterations of aquatic and terrestrial ecosystems on a local scale. \\
\hline medium & $>20 \leq 40$ & Moderate probability of alterations of aquatic and terrestrial ecosystems on a medium scale horizon \\
\hline High & $>40 \leq 60$ & Significant probability of alterations of aquatic and terrestrial ecosystems on a medium scale horizon \\
\hline Very high & $>60$ & High probability of serious alterations of aquatic and terrestrial ecosystems on a medium scale horizon \\
\hline
\end{tabular}

In a management context, the previous step is essential to establish a dialogue with managers and stakeholders, since each of the parts involved needs to accurately know what the actual state of risk is before formulating any hypothesis on management strategies. In our case, the identification of risk scenarios through risk indices allowed us to set -for each environmental compartment at stakean a priori set of reference values for the analysis of possible hazard scenarios.

\section{Analysis of Hazard Scenarios}

The design methodology for the analysis of risk scenarios is based on a joint use of various multicriteria evaluation methods. The core of the methodology is represented by the so-called Regime Analysis, extended with a complementary methodology, viz. the Flag Model. 


\subsection{Flag Model}

The main purpose of the Flag Model is to analyse whether one or more policy alternatives can be classified as acceptable or not in light of an a priori set of constraints. The model does so by comparing impact values with a set of reference values (called Critical Threshold Values). The Flag Model assesses the degree to which competing alternatives fulfil pre-defined standards or normative statements in an evaluation process (see for applications of the Flag Model [14] and [16]). The Flag Model can operate both as a classification procedure and as a visualisation method. In the former case - for example, in combination with Regime Analysis - the Flag Model can determine acceptable alternatives; accordingly, examined alternatives can then be ranked by means of Regime Analysis. In the latter case, the Flag Model can be used to visualise in an appealing way the results obtained, for example, from Regime Analysis or from other sets of classification or evaluation methods.

In this paper the Flag Model operates as a classification procedure to identify acceptable herbicide practices not posing critical risk scenarios. Acceptable alternatives are then ranked by mean of Regime Analysis. At the same time, the visualisation of the risk assessment outcomes by means of the Flag model facilitates the analysis of risk scenarios. For each risk indicators, a bandwidth of critical threshold values (CTV) is defined, which is used to set our reference system for judging the environmental impacts of alternatives ${ }^{19}$. In the present analysis, the bandwidth ranges from a maximum value (CTVmax) to a minimum value (CTVmin). For each risk index, CTVmin, CTV and CTVmax are set equal to the corresponding low, moderate and high upper bound of risk level, respectively (see Table 2). The resulting reference scheme is represented in Figure 2. Table 3 illustrates results of the Flag Analysis.

\footnotetext{
${ }^{19}$ Since in many cases experts and decision-makers may have conflicting views on the precise level of the acceptable threshold values, a bandwidth of critical threshold values - by way of sensitivity analysis - is often preferred to a single CTV.
} 
Figure 2: Reference scheme for judging alternatives within the Flag Model.

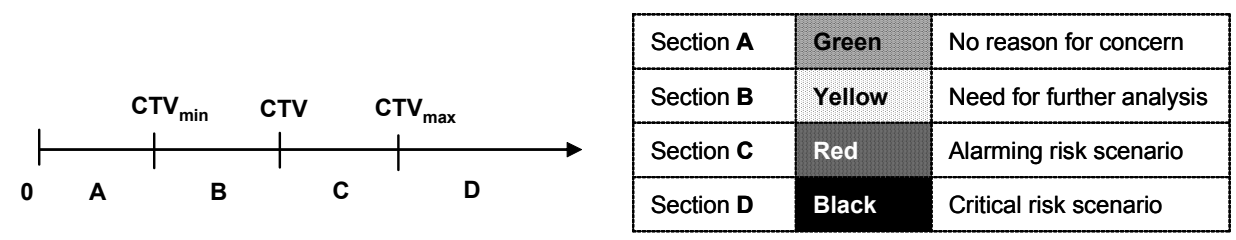

Table 3: Results of the Flag Model analysis for maize and rice.

\begin{tabular}{|c|c|c|c|c|c|c|c|c|c|c|c|c|c|}
\hline \multirow{2}{*}{$\begin{array}{c}\text { Maize } \\
\text { Risk Scenarios } \\
\end{array}$} & \multicolumn{12}{|c|}{ Alternative herbicide strategies } & \\
\hline & $A$ & & & $\mathbf{C}$ & D & $\mathbf{E}$ & $\mathbf{F}$ & $\mathbf{G}$ & $\mathbf{H}$ & I & $\mathbf{L}$ & $\mathbf{M}$ & \\
\hline Aquatic Ecosystem - Short Term & $Y$ & & & $\mathbf{Y}$ & $\mathbf{Y}$ & $\mathbf{Y}$ & G & $\mathbf{Y}$ & G & G & $\mathbf{Y}$ & $\mathbf{Y}$ & \\
\hline Aquatic Ecosystem - Long Term & $\mathbf{F}$ & & & $\mathbf{R}$ & $\mathbf{R}$ & $\mathbf{Y}$ & G & $\mathbf{Y}$ & G & G & G & $\mathbf{Y}$ & \\
\hline Terrestrial Ecosystem - Short Term & Y & & & Y & $\mathbf{Y}$ & Y & $\mathbf{Y}$ & $\mathbf{Y}$ & $\mathbf{Y}$ & $\mathbf{Y}$ & $\mathbf{Y}$ & $\mathbf{Y}$ & \\
\hline Terrestrial Ecosystem - Long Term & F & & & $\mathbf{R}$ & $\mathbf{Y}$ & G & $\mathbf{Y}$ & $\mathbf{Y}$ & $\mathbf{Y}$ & $\mathbf{Y}$ & $\mathbf{Y}$ & G & \\
\hline General Environment & $F$ & & & $\mathbf{R}$ & $\mathbf{Y}$ & $\mathbf{Y}$ & $\mathbf{Y}$ & $\mathbf{Y}$ & $\mathbf{Y}$ & $\mathbf{Y}$ & G & $\mathbf{Y}$ & \\
\hline Rice & \multicolumn{13}{|c|}{ Alternative herbicide strategies } \\
\hline Risk Scenarios & A & B & $\mathbf{C}$ & $\mathbf{D}$ & $\mathbf{E}$ & $\mathbf{F}$ & $\mathbf{G}$ & $\mathbf{H}$ & $\mathbf{I}$ & $\mathbf{L}$ & $\mathbf{M}$ & $\mathbf{N}$ & $\mathrm{O}$ \\
\hline Aquatic Ecosystem - Short Term & $\mathbf{R}$ & $\mathbf{Y}$ & $\mathbf{R}$ & $\mathbf{R}$ & $\mathbf{R}$ & $Y$ & $\mathbf{R}$ & $\mathbf{Y}$ & $\mathbf{Y}$ & $\mathbf{Y}$ & $\mathbf{Y}$ & $\mathbf{Y}$ & $\mathbf{Y}$ \\
\hline Aquatic Ecosystem - Long Term & $\mathbf{R}$ & $\mathbf{Y}$ & $\mathbf{R}$ & $\mathbf{R}$ & B & B & $\mathbf{R}$ & $\mathbf{Y}$ & $\mathbf{Y}$ & Y & $\mathbf{Y}$ & $\mathbf{Y}$ & $\mathbf{Y}$ \\
\hline Terrestrial Ecosystem - Short Term & $\mathbf{Y}$ & $\mathbf{Y}$ & Y & $\mathbf{Y}$ & B & B & Y & Y & $\mathbf{Y}$ & Y & $\mathbf{R}$ & Y & $\mathbf{Y}$ \\
\hline Terrestrial Ecosystem - Long Term & $\mathbf{Y}$ & $\mathbf{Y}$ & Y & $\mathbf{Y}$ & $\mathbf{R}$ & $\mathbf{R}$ & $\mathbf{Y}$ & $\mathbf{Y}$ & $\mathbf{Y}$ & Y & $\mathbf{Y}$ & $\mathbf{Y}$ & G \\
\hline General Environment & $\mathbf{Y}$ & $\mathbf{Y}$ & $\mathbf{R}$ & $\mathbf{Y}$ & $\mathbf{R}$ & $\mathbf{R}$ & $\mathbf{Y}$ & $\mathbf{Y}$ & $\mathbf{Y}$ & $\mathbf{Y}$ & $\mathbf{Y}$ & $\mathbf{Y}$ & $\mathbf{Y}$ \\
\hline
\end{tabular}

For the maize production, the Flag Analysis of risk scenarios provides quite encouraging results, and none of the alternatives gets a black flag. The graphical visualisation suggests that alternative herbicide strategies can be grouped into two major sets posing comparable risks to non-target agricultural ecosystems. In particular, the alternatives from A to D are related to more severe risk scenarios (yellow or red flags), whereas the remaining alternatives get yellow or green flags. Main differences refer to long-term risk scenarios, both for aquatic and terrestrial ecosystems, which can be trace back to the higher persistency of the herbicide active ingredients used in treatments A, B, C and D. It is interesting to notice that such subgroups correspond, exactly, to pre-emergence and post-emergence strategies, respectively ${ }^{20}$. The case of rice is slightly more complex. Two strategies -E and F- present critical risk scenarios associated to red or black flags both for the aquatic and the 
terrestrial ecosystems and are therefore excluded from the set of possible treatments for rice.

Alternatives A, C, D, and $\mathrm{G}$ are associated to alarming risk scenarios for the surface water environment; whereas, overall, the remaining strategies seem to be more environmental friendly.

\subsection{Regime Analysis}

The risk analysis is finally enriched within a MCA frame which integrates environmental, agronomic and economic objectives. Multi-criteria analysis comprises various classes of decisionmaking approaches. The multi-assessment method used in our methodology is Regime Analysis, a discrete multi-assessment method (for details see [15]). Regime is a generalised form of concordance analysis, based in essence on a generalisation of pairwise comparison methods. The fundamental framework of the method is based upon two kinds of input data: an impact matrix (structured information table) and a set of (politically determined) weights (for details we recommend: [11] and [15]). The impact matrix is composed of elements that measure the effect of each considered alternative in relation to each policy relevant criterion. The set of weights incorporates information concerning the relative importance of the criteria in the evaluation. In case there is no prioritisation of criteria in the evaluation process, all criteria will be assigned the same numerical weight value.

In our analysis, Regime is applied separately to each of two sets of acceptable alternative herbicide strategies for maize and rice production. The relevant criteria include short-term and longterm risks for non-target ecosystems, agronomic efficacy ${ }^{21}$ and the cost of herbicide alternatives. The impact matrixes are presented in Table 4. We compare results obtained using two different weight vectors: one is seeking for the higher level of environmental protection; the other strongly privileges the agronomic performance (see

\footnotetext{
${ }^{20}$ Pre-emergence and post emergence herbicides are applied before and after the outgrowth of the plant, respectively.
} 
Figure 3, Figure 4). In particular, the 'environmental' weight vector favours the long-term environmental sustainability against the cost of the herbicide treatment; nevertheless, the agricultural efficacy is still considered a relevant decision criterion. The 'agricultural performance' vector, by contrast, is unfavourable to expensive and/or inefficient herbicide strategies ${ }^{22}$.

Table 4: Impact matrix of alternative herbicide treatments for maize and rice crops, respectively. For each decision criteria we report values before standardization.

\begin{tabular}{l|ccccccccccc}
\hline \multicolumn{1}{c}{ CRITERIA $(*)$} & \multicolumn{10}{c}{ MAIZE: Alternative Herbicide Strategies } \\
\cline { 2 - 11 } & A & B & C & D & E & F & G & H & I & L & M \\
\hline Cost of the treatment & 57,8 & 56,8 & 75,9 & 54,2 & 81,1 & 94,5 & 72,3 & 90,9 & 90,6 & 118,5 & 77,2 \\
Agronomic Efficacy - Grass weed & 10,0 & 10,0 & 10,0 & 8,0 & 9,2 & 9,2 & 9,4 & 9,2 & 9,7 & 9,7 & 9,7 \\
Agronomic Efficacy - Dicotyledon weed & 9,5 & 9,2 & 8,3 & 8,8 & 9,2 & 9,8 & 9,6 & 8,5 & 9,9 & 9,7 & 9,4 \\
Risk for Aquatic Ecosystem - Short Term & 21,5 & 17,5 & 27,0 & 17,5 & 6,0 & 0,0 & 17,5 & 0,0 & 0,0 & 24,0 & 6,0 \\
Risk for Aquatic Ecosystem - Long Term & 56,3 & 41,2 & 58,8 & 39,0 & 7,5 & 2,1 & 23,7 & 1,8 & 3,6 & 3,5 & 9,0 \\
Risk for Terrestrial Ecosystem - Short Term & 19,0 & 28,0 & 31,0 & 20,5 & 6,0 & 33,0 & 36,0 & 35,0 & 39,0 & 35,5 & 9,0 \\
Risk for Terrestrial Ecosystem - Long Term & 48,3 & 48,4 & 51,6 & 20,9 & 5,0 & 9,4 & 25,2 & 6,8 & 9,8 & 6,0 & 5,4 \\
Risk for General Environment & 45,2 & 40,2 & 53,6 & 36,6 & 15,9 & 11,2 & 21,3 & 16,2 & 12,0 & 7,4 & 16,7 \\
\hline
\end{tabular}

\begin{tabular}{|c|c|c|c|c|c|c|c|c|c|c|c|c|c|}
\hline \multirow{2}{*}{ CRITERIA $(*)$} & \multicolumn{13}{|c|}{ RICE: Alternative Herbicide Strategies } \\
\hline & A & B & C & D & $\mathrm{E}$ & $\mathrm{F}$ & $\mathbf{G}$ & $\mathrm{H}$ & 1 & $\mathrm{~L}$ & M & $\mathbf{N}$ & $\mathbf{0}$ \\
\hline Cost of the treatment & 149,3 & 117,8 & 67,1 & 132,2 & 127,6 & 51,1 & 126,5 & 130,2 & 85,2 & 198,3 & 43,6 & 39,3 & 124,0 \\
\hline Agronomic Efficacy - Dicotyledon weed & 10,0 & 10,0 & 10,0 & 10,0 & 10,0 & 10,0 & 10,0 & 7,0 & 10,0 & 10,0 & 8,0 & 9,0 & 8,5 \\
\hline Risk for Aquatic Ecosystem - Short Term & 43,0 & 17,5 & 43,0 & 51,0 & 51,0 & 39,0 & 54,0 & 24,0 & 39,0 & 5,5 & 29,5 & 29,5 & 12,0 \\
\hline Risk for Terrestrial Ecosystem - Short Term & 30,0 & 36,0 & 36,0 & 39,0 & 73,0 & 72,0 & 40,0 & 19,5 & 47,0 & 35,0 & 53,5 & 29,0 & 7,0 \\
\hline Risk for Terrestrial Ecosystem - Long Term & 27,0 & 18,0 & 39,4 & 36,0 & 57,4 & 52,0 & 21,4 & 10,8 & 16,0 & 18,8 & 20,5 & 14,0 & 3,2 \\
\hline Risk for General Environment & 20,6 & 17,1 & 42,1 & 25,8 & 50,8 & 46,1 & 25,0 & 14,7 & 20,3 & 11,3 & 27,2 & 23,1 & 12,3 \\
\hline
\end{tabular}

(*) Cost of the treatment expressed in [Euros/ha]; Agronomic efficacy can assume values ranging from 4 to 10; Risk indicators can assume values ranging from 0 to 100 .

\footnotetext{
${ }^{21}$ In particular, we consider the agronomic efficacy of alternative herbicide treatments against grass weed and dicotyledonous weed. The data employed in the analysis refer to the potential agronomic efficacy of different chemical weed control strategies, as evaluated in field experiments ([20], [21], [22] and [23]). For rice, in particular, the agronomic efficacy against grass weeds refers to Echinochloa spp., one of the most aggressive weed in rice production.

${ }^{22}$ The 'environmental' vector assigns 4 and 3 to the long-term and short-term risk indicators, respectively; 2 to the agronomic efficacy criteria and 1 to the cost criterion. The 'agricultural performance' vector, by contrast, assigns 4 to the agronomic efficacy criteria, 3 to the cost criterion, 2 and 1 , to the long-term and short-term risk indicators, respectively.
} 
Table 5: Retail data and spread of alternative weed control strategies employed in maize production (1999).

\begin{tabular}{|c|c|c|c|}
\hline \multicolumn{2}{|r|}{ Alternative herbicide practices } & \multicolumn{2}{|c|}{$\%$ Area $\left({ }^{\circ}\right)$} \\
\hline 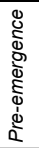 & $\begin{array}{l}\text { A. } \text { Alachlor + Terbutilazine } \\
\text { B. Metolachlor + Terbutilazine } \\
\text { C. Metolachlor + Pendimetalin } \\
\text { D. Isoxaflutol + Aclonifen }\end{array}$ & $\begin{array}{c}16,4 \\
22,7 \\
4,5 \\
21,8\end{array}$ & 65,5 \\
\hline $\begin{array}{l}\searrow \\
0 \\
\Phi \\
\Phi \\
\Phi \\
\Phi \\
\frac{1}{\infty} \\
0 \\
0\end{array}$ & $\begin{array}{l}\text { E. Rimsulfuron + Prosulfuron + Primisulfuron } \\
\text { F. Rimsulfuron + Dicamba } \\
\text { G. Rimsulfuron + Terbutilazine } \\
\text { H. Rimsulfuron + Fluroxipir } \\
\text { I. Nicosulfuron + Dicamba } \\
\text { L. Nicosulfuron + Sulcotrione } \\
\text { M. Nicosulfuron + Prosulfuron + Primisulfuron }\end{array}$ & $\begin{array}{l}1,4 \\
8,2 \\
0,9 \\
0,5 \\
5,5 \\
3,6 \\
1,4\end{array}$ & 21,4 \\
\hline
\end{tabular}

$\left(^{\circ}\right)$ In Italy, 1,100,000 ha of the total cultivated area under maize crop. The $70 \%$ is treated with pre-emergence herbicides; whereas the remaining $30 \%$ is treated with some post-emergence strategies.

Figure 3: Results of Regime Analysis for maize. Alternatives' ranking obtained applying the 'environmental' and the 'agronomic performance' weight vector.
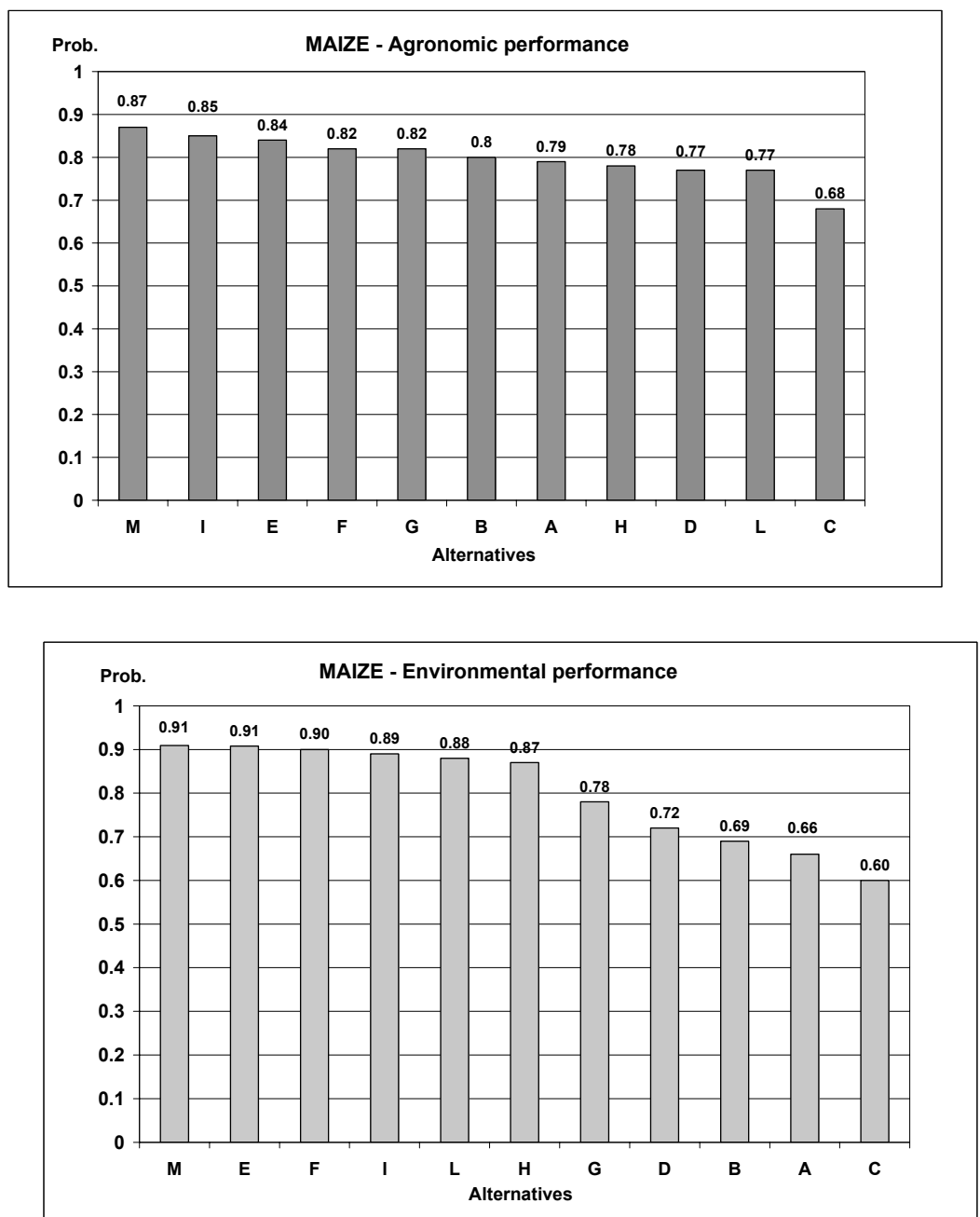
Figure 4: Results of Regime Analysis for rice. Alternatives' ranking obtained applying the 'environmental' and the 'agronomic performance' weight vector.
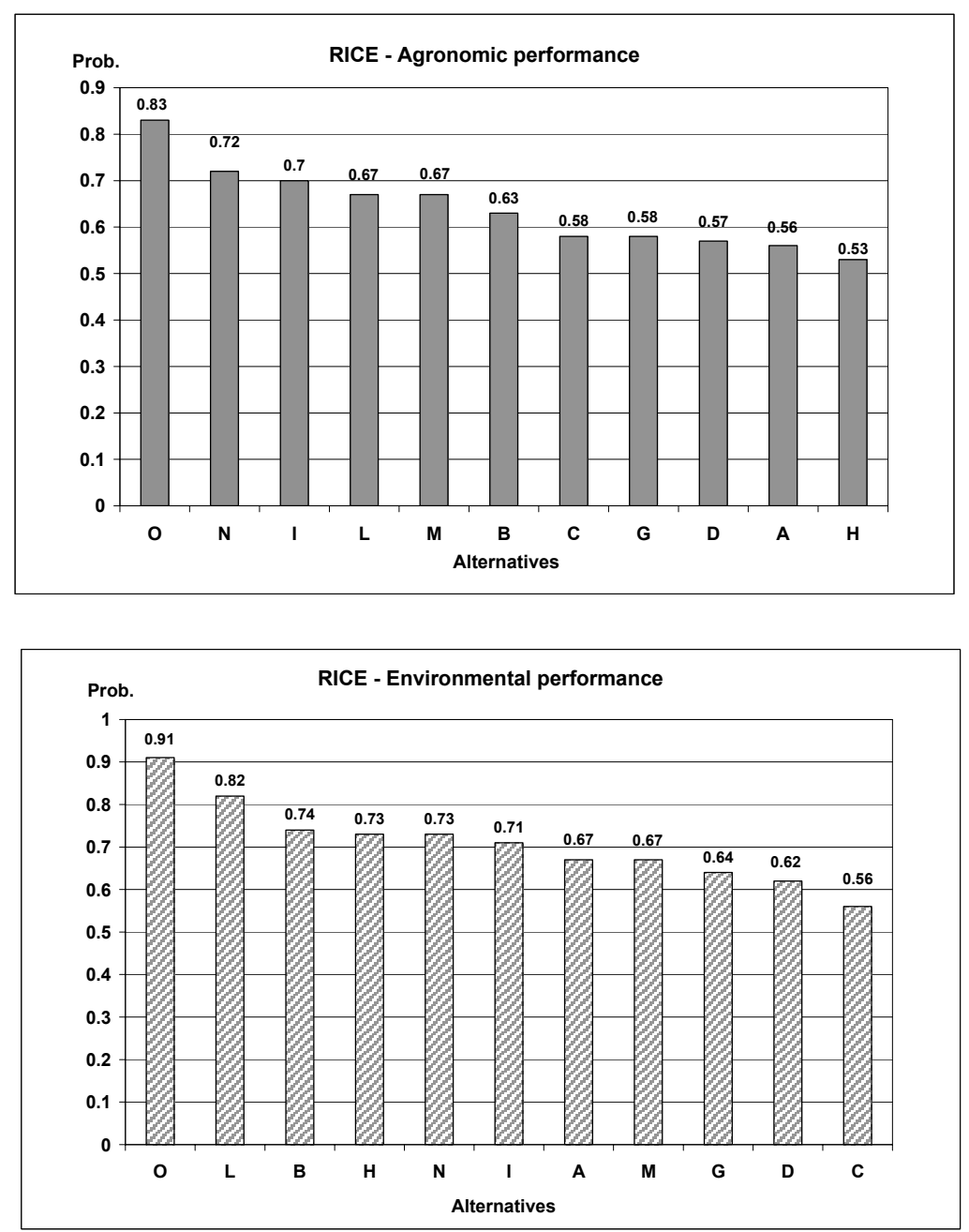

Figure 5: Results of Regime Analysis for rice, considering all the initial herbicide alternatives. Alternatives' ranking obtained applying the 'environmental' and the 'agronomic performance' weight vector.

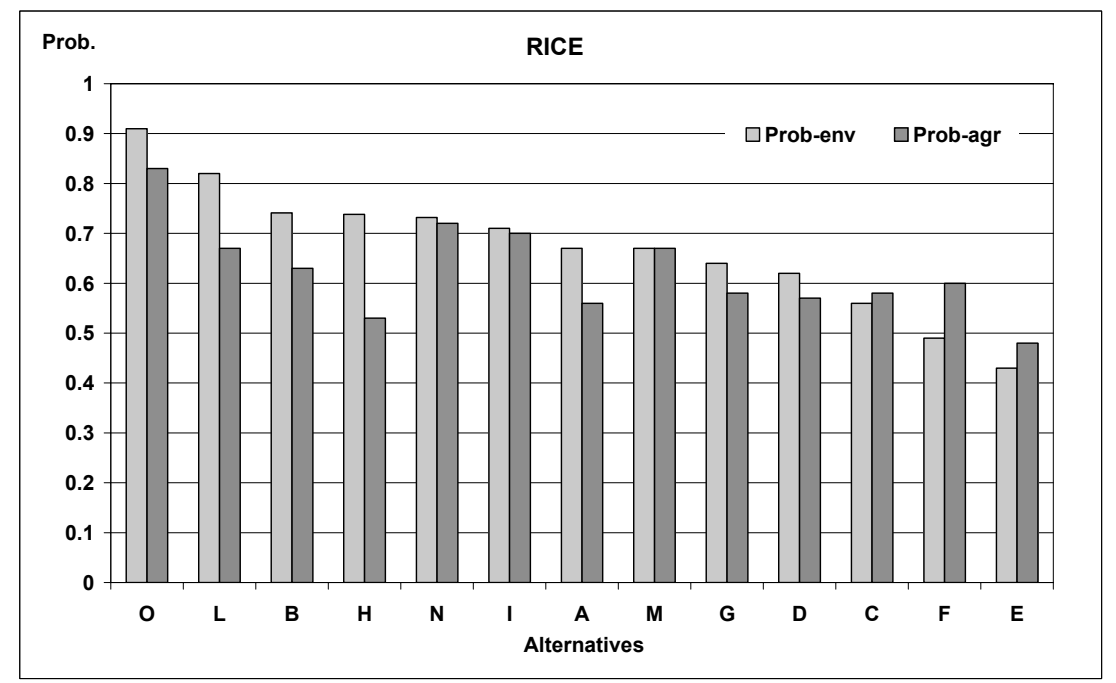


A closer look to the results (

Figure 3, Figure 4) shows that the ranking of alternatives obtained using the two aforementioned decision perspectives are quite close to each other, especially in the case of maize production. Both for maize and rice, the best herbicide strategy does not change as the weight vector changes; this suggests that the first ranked alternative satisfies both the environment and agriculture-driven perspectives. For maize, even the following three alternatives remain unchanged, although their relative order is different. Some larger variations can be noticed in the last positions of the ranking; however, the whole variation of the decision scores does not exceed 0,3 points. For rice, the decision scores show a slightly higher variation which reaches 0,35 -using the environmental weighting vector- and 0,29 using the second weigh vector. Consistently with the results of the Flag Model for rice, if the two rejected alternatives-E and F- are included into the analysis, they get indeed the two lowest scores in the 'environmental' ranking. However, in the 'agricultural' rankingalternative $\mathrm{F}$ is strongly privileged thanks to a high agronomic efficacy and a low cost (Figure 5).

To test the usefulness of this type of analysis for management purposes, let us take a closer look at the Italian herbicide market and see whether our best alternatives correspond to what is actually used by the Italian farmers. The case of maize is particularly interesting; Table 5 shows the retail data of alternative herbicide treatments for the maize production (1999). As one can notice, Italian farmers strongly prefer adopting pre-emergence herbicide treatments (options from A to D) in respect to post-emergence ones (alternatives from $\mathrm{E}$ to $\mathrm{M}$ ). The reason of such preference is easily imputable to the lower cost of pre-emergence alternatives and, secondly, to a slightly stronger agronomic performance compared to post-emergence actions. The actual situation of the herbicide practices in Italy, therefore, is in contrast with what -in our analysis- emerges as the herbicide 
options to be adopted towards the gratification of basic economic, agronomic and environmental criteria. Alternative M, for instance, which gets the highest decision score using both the weighting procedures, is used to treat only the $1.4 \%$ of the total area under corn cultivation. By contrast, alternative B, employed on the $22.7 \%$ of the total area under maize, is placed at the bottom of the Regime ranking; even when embracing a more agricultural-driven perspective it is surpassed by a number of other alternatives.

\section{Discussion}

The policy issue here is how best to use scientific information on the environmental risk of pesticide use to lower the impact of conventional agricultural practices. In particular, a more immediate issue is how to combine and analyse the available information for useful management actions.

One aim of this paper was to draw attention on the design of pesticide risk indicators as innovative risk management instruments. The synthesis of information on pesticide hazard and exposure into risk indices is found to be useful for providing plausible visions on the status quo of pesticide risks and to identify potential trouble spots where risk reduction might be a main concern. The inclusion in the analysis of a set of indicators representing pesticide hazards along a number of ecological dimensions is also found to be important for articulating trade-offs in management objectives across different environmental concerns. Besides, our empirical analysis confirms that multicriteria techniques constitute a suitable framework to apply risk indices as decision support tools.

Overall, this study suggests the potential of risk indicators to support the definition of risk management priorities. In this sense, a major contribution of risk indicators is to allow the acquisition of information of primary importance, in an easily interpretable format and at a relative moderate cost. The level of detail of such tools, either compound or mixture-specific, is indeed very high compared to the cost involved in the design and implementation of this kind of approach. 
Nevertheless, one should be aware that the cost-effectiveness of an indicator depends upon both the purpose one want to mach and how it will be deployed. Only from this perspective, therefore, it is relevant to express satisfaction or discontent for the quality of results.

Our tentative empirical application suggests that -whenever the analysis aims at giving preliminary insights on severity and dimension of environmental risks- risk indicators based on standardised worst-case scenarios have relevant management potential. Yet, as the analysis gets site-specific and requires direct connection to the territory, the potential of such tools decrease since risk scenarios need to incorporate the environmental variability of local or regional boundary conditions. Finally, whenever the study aims at monitoring risk trends over time and space -kept data on toxicity and exposure at a lower level of detail- integrate data on conditions and quantity of pesticide use are requisite.

Yet, many research challenges remain open. In particular, efforts are needed to clarify the basic scientific requisites necessary to design risk indices, given different management tasks. In this sense, a close cooperation among scientists and managers is advocated to finalise the indices' design procedure, thus increasing its cost-effectiveness and implementation potential. 


\section{Acknowledgements}

Section 1 to 2 can be attributed jointly to Chiara Travisi and Paolo Giacomelli; Section 3 and 4 to Chiara Travisi and Marco Vigni, while Section 5 and 6 involved jointly Chiara Travisi and Peter

Nijkamp. The authors wish to thank Dr. Antonio Finizio for the development and application of the pesticide indices used here.

\section{References}

1. Blamey, R.K., Common, M.S. (2000). "Valuation and ethics in environmental economics". In van den Bergh, J.C.J.M. (Ed.), Handbook of Environmental and Resource Economics, Edward Elgar, Cheltenham, U.K.

2. Brethour, C. A., Weersink, A. (2001). "An Economic evaluation of the environmental benefits from pesticide reduction", Agricultural Economics, 25: 219-226.

3. Cuyno, L. C. M., Norton, G. W., Rola A. (2001). "Economic analysis of environmental benefits of integrated pest management: a Philippine case study", Agricultural Economics, 25: 227-233.

4. Di Guardo, A., Calamari, D., Zanin, G., Consalter, A., Mackay, D. (1994). "A fugacity model of pesticide runoff to surface water, developement and valication", Chemosphere, 28: 511-531.

5. European and Mediterranean Plant Protection Organisation Council of Europe (EPPO) (1993). Decision-making scheme for the environmental risk assessment of plant protection products. Bull. OEPP/EPPO 23: 151-165.

6. European and Mediterranean Plant Protection Organisation Council of Europe (EPPO) (1994a). Decision-making scheme for the environmental risk assessment of plant protection products. Bull. OEPP/EPPO 23: 17-35.

7. European and Mediterranean Plant Protection Organisation Council of Europe (EPPO) (1994b). Decision-making scheme for the environmental risk assessment of plant protection products. Bull. OEPP/EPPO 23: 37-87.

8. Faasen, R. (1994). “Agricultural pesticide use: a threat to the European environment?", Proceeding of the workshop on a framework for the sustainable use of plant protection products in the European Union (Brussels 14-15 June 1994), DHV Environment and Infrastructure, Amersfoort.

9. Finizio, A. (1999a). "The Environmental impact of pesticides. Risk evaluation for non target organisms" (L'impatto ambientale dei prodotti fotosanitari. Valutazione del rischio per gli organismi non bersaglio). Quaderni tecnici dell'Agenzia Nazionale per la Protezione dell'Ambiente (ANPA) N10-I.

10. Finizio, A. (1999b). "The Environmental impact of pesticides. Risk evaluation for non target organisms" (L'impatto ambientale dei prodotti fotosanitari. Valutazione del rischio per gli organismi non bersaglio). Quaderni tecnici dell'Agenzia Nazionale per la Protezione dell'Ambiente (ANPA) N10-II.

11. Finizio, A., Calliera, M., Vighi, M. (2001). "Rating Systems for pesticide risk classification on different ecosystems", Ecotoxicology and Environmental Safety, 49: 262-274.

12. Foster V., Mourato, S. (2000). Valuing the multiple impacts of pesticides use in the UK: A Contingent Ranking Approach, Journal of Agricultural Economics, 51: 1-21.

13. Fu T-T, Liu J-T, Hammitt, J.K. (1999). „Consumer willingness to pay for low-pesticide fresh produce in Taiwan”, Journal of Agricultural Economics, 50(2): 220-233.

14. Hermanides, G. and Nijkamp, P. (1998). "Multicriteria evaluation of sustainable agricultural land use: a case study of Lesvos", in Beinat E. and Nijkamp P. (Eds.): Multicriteria Analysis for Land Use Management, Kluver, Dordrecht, 1998, 61-78.

15. Greco W., Unkelbach H.D., Pöch G., Suhnel J., Kundi M., Bodeker W. (1992). “Consensus on concepts and terminology for combined-action assessment: the Saariselkä agreement”, Arch. Complex Environ. Stud. 4 (3): 65-72.

16. Hinloopen, E., Nijkamp, P., Rietveld, P. (1983). "Qualitative discrete multiple criteria choice models", Regional Planning, Regional Science and Urban Economics, 77-102. 
17. Kovach, J., Petzold, C., Degni, J., and Tette, J. (1992). "A method to measure the environmental effects of pesticides", N.Y. Food Life Scientific Bullettine, N 139.

18. Mackay, D. (1991). Multimedia Environmental Models: The Fugacity Approach, Lewis Publishers.

19. Nijkamp, P. and Ouwersloot, H. (1998). "A decision support system for regional sustainable development: the flag model”, in J.C.J.M. van den Bergh and M.W. Hofkes (Eds.): Theory and Implementation of Sustainable Development Modeling, Kluwer, Dordrecht, 1998, 255-273.

20. Nijkamp, P., Rietveld, P., Voogd, H. (1990). Multicriteria Analysis for Physical Planning, Elsevier, Amsterdam.

21. Nijkamp, P., Vreeker, R. (2000). "Sustainability assessment of development scenarios: methodology and application to Thailan”, Ecological Economics, 33: 7-27.

22. OECD (1996). "Activities to reduce pesticide risks in OECD and Selected FAO Countries", Part I: Summary Report. OECD Environmental Health and Safety Publications, Series on Pesticides N. 4, Paris.

23. OECD (1997). Report of the OECD Workshop on Pesticide Risk Indicators (Copenahgen, 21-23 April 1997), http://www.oecd.org/pest/pestrr/cop_fin.doc

24. OECD (1999). Report of the Second OECD Workshop on Pesticide Risk Indicators (Braunschweig, Germany, 1-3 June 1999), http://www.oecd.org/riskind/report4.doc

25. Rapparini, G. (1998a). "Il diserbo del riso", L'informatore agrario, 13: 69-79.

26. Rapparini, G. (1999a). "Il diserbo di post-emergenza dei cereali vernini”, L'informatore agrario, 2: 65-77.

27. Rapparini, G. (1999b). "Il diserbo del mais e del sorgo", L'informatore agrario, 8: 65-82.

28. Rapparini, G.-Bartolini, D.-Campagna, G. (1998b). "Verifiche sperimentali sul diserbo chimico del mais", L'informatore agrario, 19: 67-75.

29. Sampaolo, A., Binetti, R. (1986). "Elaboration of a practical method for priority selection and risk assessment among existing chemicals", Regulatory Toxicology and Pharmacology, 129-154.

30. Sbriscia Fioretti, C.-Zanin, G.-Ferrario, P-Vighi, M. (1998). "Chemical characteristics: the case of herbicides in Italy", in Swanson, T., Vighi, M. (Eds.): Regulating Chemical Accumulation in the Environment. The Integration of Toxicology and Economics in Environmental Policy-making, Cambridge, Cambridge University Press, pp.23-49. 